\title{
Soluble IL6R represents a miR-34a target: potential implications for the recently identified IL-6R/STAT3/miR-34a feed-back loop
}

\author{
Huihui Li1,*, Matjaz Rokavec ${ }^{1, *}$ and Heiko Hermeking ${ }^{1,2,3}$ \\ ${ }^{1}$ Experimental and Molecular Pathology, Institute of Pathology, Ludwig-Maximilians-Universität München, Munich, Germany \\ ${ }^{2}$ German Cancer Consortium (DKTK), Heidelberg, Germany \\ ${ }^{3}$ German Cancer Research Center (DKFZ), Heidelberg, Germany \\ * These authors have contributed equally to this work
}

Correspondence to: Heiko Hermeking, email: heiko.hermeking@med.uni-muenchen.de Keywords: miR-34a, colorectal cancer, IL-6R, STAT3, inflammation

Received: May 04, $2015 \quad$ Accepted: May 24, $2015 \quad$ Published: June 02, 2015

This is an open-access article distributed under the terms of the Creative Commons Attribution License, which permits unrestricted use, distribution, and reproduction in any medium, provided the original author and source are credited.

\section{ABSTRACT}

We previously reported that IL-6R, STAT3 and miR-34a form a positive feedbackloop, which promotes epithelial to mesenchymal transition (EMT), invasion, and metastasis of colorectal cancer (CRC) [1]. In that study only the membrane-bound form of the IL-6R was shown to be repressed by miR-34a. Here, we show that also the mRNA encoding the soluble $I L 6 R(s-I L-6 R)$ is directly targeted and repressed by miR$34 a$. Accordingly, the concentration of $S-I L 6 R$ protein was decreased in conditioned media of CRC cell lines ectopically expressing miR-34a. The s-IL-6R mediates IL-6 trans-signaling, which also affects cells that do not express the IL-6R. Since IL-6 trans-signaling is involved in numerous inflammatory disease states these findings may be relevant for future therapeutic approaches.

\section{INTRODUCTION}

Signaling mediated via the interleukin-6 (IL-6)/ interleukin-6 receptor (IL-6R) plays a pivotal role during immune responses and in cancer [2-4]. Upon binding of IL-6, IL-6R associates with gp130, which initiates intracellular signal transduction via the JAK/STAT and the Ras/MAPK/AKT pathways [5]. Besides classical IL-6 signaling, which involves the membrane-bound IL-6R (m-IL-6R), also IL-6 trans-signaling has been described [6]. The later involves the s-IL-6R, which is shedded/ released by cells and in complex with IL-6 binds to and activates gp130. Thereby, IL-6 trans-signaling can affect cells that do not express the IL-6R [7]. The human s-IL$6 \mathrm{R}$ protein is either generated by skipping exon 9 via alternative splicing and subsequent earlier termination of translation caused by a stop codon in the alternative reading frame in exon 10 (Figure 1A) or by cleavage of the m-IL-6R protein by the $\alpha$-secretase ADAM17 upstream of the transmembrane (TM) domain (Figure 1B) [8]. The N-terminal IL-6R fragment is shedded, whereas the C-terminal fragment is cleaved by $\gamma$-secretase in the transmembrane (TM) domain and removed by lysosomal degradation.
The genes encoding the microRNAs (miRNAs) miR-34a and miR-34b/c are direct p53 target genes and mediate tumor suppressive effects of p53 (reviewed in $[9,10]) . \mathrm{miR}-34 \mathrm{a} / \mathrm{b} / \mathrm{c}$ have been shown to inhibit cell cycle progression, angiogenesis, stemness, invasion and metastasis by directly down-regulating the expression of key factors promoting these processes. For example, the direct repression of the EMT-TF SNAIL contributes to mesenchymal-epithelial transition induced by p53 [11]. Also Cyclin E, SIRT1, MYC and Wnt/TCF7, Yin Yang 1, L1CAM and c-Kit have been shown to represent direct miR-34 targets, which mediate its tumor suppressive effects [12-18]. Furthermore, miR-34 mimetics are currently being tested for their therapeutic value in a phase 1 trial [19]. In addition, the inactivation of $m i R-34 a$ by $\mathrm{CpG}$ methylation may be used for prognostic purposes [20].

Recently, we showed that m-IL-6R is part of a positive feedback loop involving miR-34a and STAT3 [1], which was commented on in [21]. We identified the $I L 6 R$ mRNA as a direct target of miR-34a and showed that $m i R-34 a$ is directly repressed by STAT3. The activation of this loop was required for EMT, invasion, and metastasis and is associated with nodal and distant metastasis in 
colorectal cancer $(\mathrm{CRC})$ patients.

\section{RESULTS}

Here we asked whether the s-IL-6R is a direct target of miR-34a. Based on Ensembl, the s-IL6R mRNA isoform has a shorter 3'-UTR sequence than the m-IL6R isoform. Yet, it contains a miR-34a seed-matching site, which corresponds to the first site in the m-IL6R encoding mRNA (Figure 2A). We found that ectopic miR-34a resulted in repression of a s-IL6R 3'-UTR reporter in a dual reporter assay (Figure 2B). This repression was alleviated by mutation of the miR-34a seed-matching site (Figure 2B). To investigate whether also the endogenous transcript isoform that encodes $s-I L-6 R$ is regulated by miR-34a, we designed qPCR primers that exclusively recognize the $m-I L-6 R$ or the $s-I L 6 R$ mRNA isoform (Figure 3A). Indeed, ectopic expression of miR-34a resulted in a decrease of the membrane-bound and soluble IL6R isoforms in SW480 cells (Figure 3B). Next, we determined the effect of ectopic miR-34a expression on S-IL-6R protein expression by transfecting SW480 and SW620 cells with pre-miR-34a oligonucleotides. s-IL6R specific ELISA analyses showed that in both cell lines ectopic expression of miR-34a significantly decreased the concentration of s-IL-6R in conditioned media (Figure 3C, 3D). Finally, we showed that the levels of s-IL-6R are elevated in the conditioned media of mesenchymallike SW480 and SW620 CRC cells that express low levels of miR-34a, whereas epithelial DLD1, HCT15, HT29, CACO2, and LST174 cells express high levels of miR-34a (Figure 3E, 3F). These latter results are in agreement with a previous publication showing that SW620 cells secrete more s-IL-6R than DLD1 and HT29 cells [22].

In addition, it should be mentioned that the NCBI GenBank lists an additional IL6R isoform that lacks exon 9, which encodes the transmembrane domain
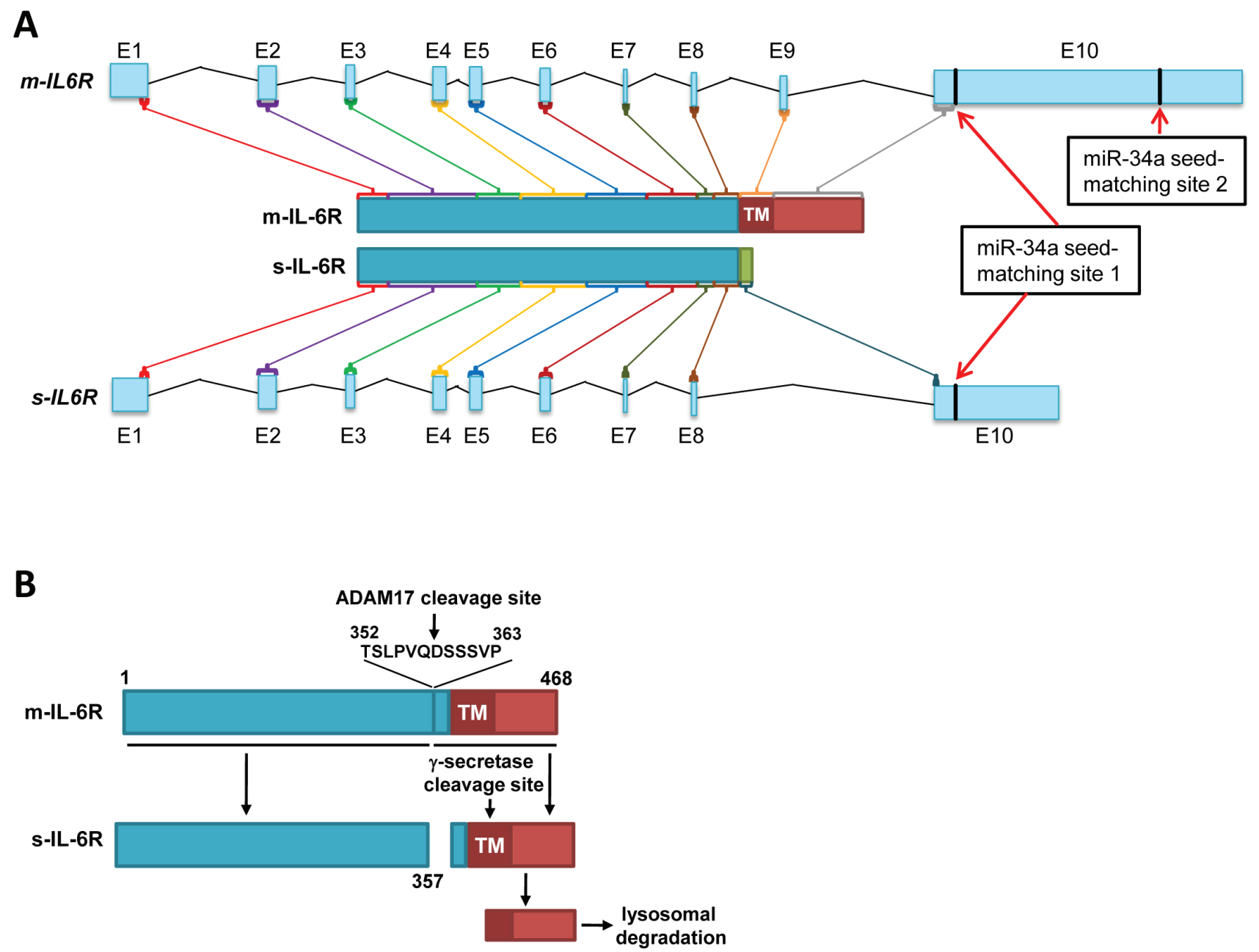

Figure 1: Generation of soluble and membrane IL-6R mRNA and protein isoforms. A. Generation of IL-6R protein isoforms by alternative splicing. The organization of two alternatively spliced human IL6R mRNA products and the resulting proteins is depicted. Protein-coding ORFs are indicated by different colors and are shown as final protein products in the center. The miR-34a seed-matching sequences are indicated with red arrows. The scheme is based on data from Ensembl (release 78 - December 2014), Genome assembly: GRCh38 (Homo sapiens). B. Generation of soluble IL-6R by cleavage of m-IL-6R protein. 
(NM_181359.2). The s-IL6R mRNA isoform also lacks exon 9. Thus, the NM_181359.2 mRNA may also encode the $s-I L-6 R$ protein. However, this isoform contains the full length 3'-UTR and therefore the two miR-34a binding sites as the m-IL6R isoform, which we have shown to mediate direct repression of IL6R by miR-34a [1].

\section{DISCUSSION}

IL-6 trans-signaling, which is mediated via the s-IL-6R, is critically involved in several inflammatory and autoimmune diseases including inflammatory bowel disease, rheumatoid arthritis, and asthma, as well as colitis-associated cancer [23]. Therefore, understanding the regulation of IL- 6 trans-signaling could represent a basis for development of novel drugs that might be useful for treatment of several diseases. Recently, we identified the m-IL-6R as a direct target of miR-34a [1]. Here we extend our previous findings and demonstrate that also the s-IL-6R is a direct target of miR-34a. We show that not only the s-IL-6R that is generated by proteolytic cleavage of $\mathrm{m}-\mathrm{IL}-6 \mathrm{R}$, but also the s-IL-6R that is produced by

A
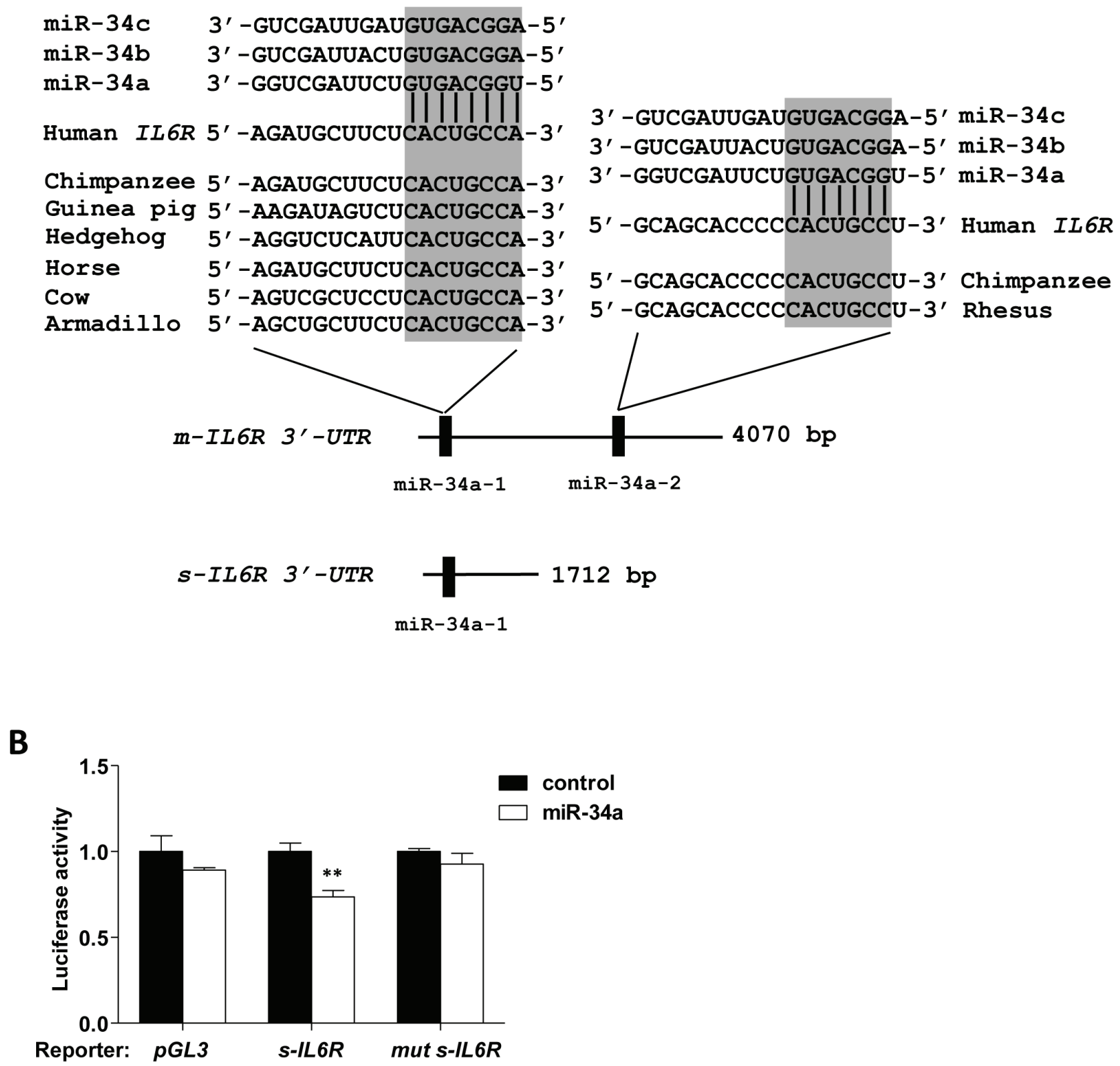

Figure 2: Direct regulation of $\boldsymbol{s}-\boldsymbol{I L} 6 \boldsymbol{R}$ by miR-34a. A. Schematic representation of the 3'-UTRs of human membrane-bound and soluble $I L 6 R$ mRNA isoforms indicating the miR-34 seed-matching sequences and their phylogenetic conservation. B. Dual reporter assay after transfection of H1299 cells with pre-miR-34a oligonucleotides and human s-IL6R 3'-UTR reporter constructs. 
A

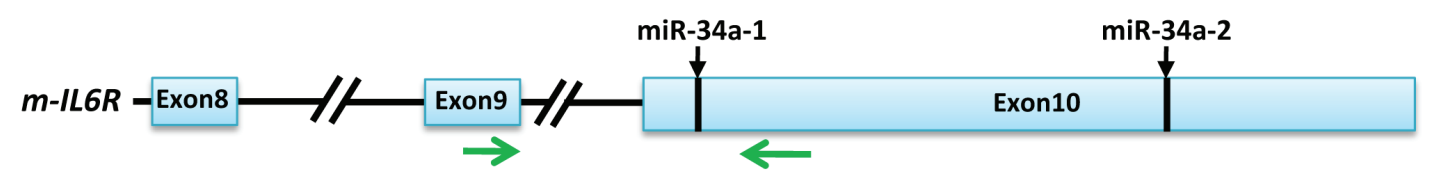

qPCR primers specific for $m$-IL6R
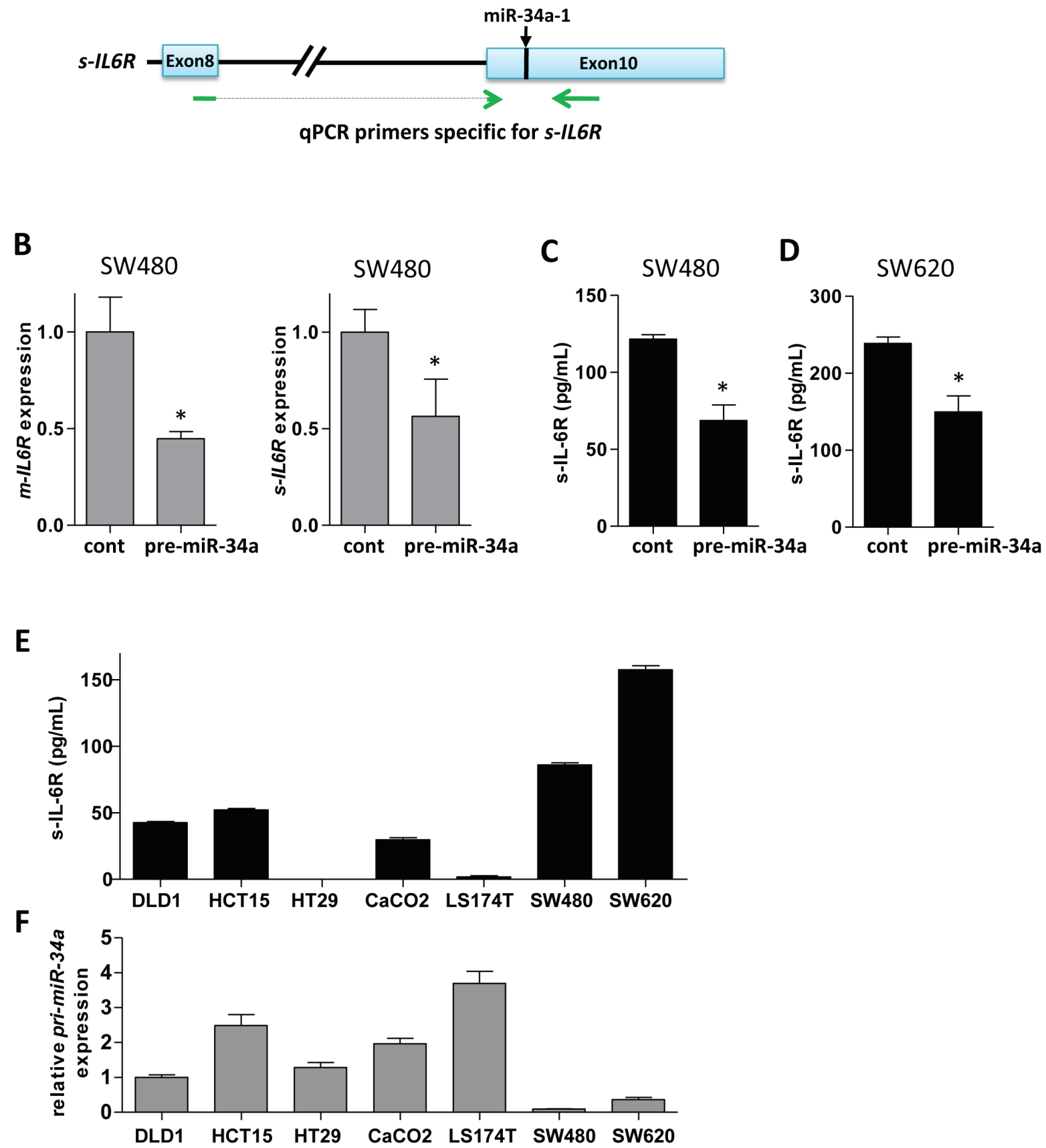

Figure 3: miR-34a down-regulates s-IL-6R expression in CRC cell lines. A. Location of qPCR primers specific for mRNA isoforms encoding membrane-bound and soluble $I L 6 R$, respectively. Exon 9 encodes the transmembrane domain, which is absent in the soluble $I L 6 R$ isoform. miR-34a seed-matching sites are indicated. B. Expression of m- and s- $I L 6 R$ mRNA in SW480 cells transfected with control or pre-miR-34a oligonucleotides. C., D. Expression of s-IL-6R protein in the indicated cell lines transfected with control or pre-miR-34a oligonucleotides. After 72 hours medium was changed and 24 hours later conditioned media was subjected to ELISA. E. Expression of s-IL-6R protein in indicated cell lines. Equal numbers of cells was seeded and conditioned media was analyzed by s-IL-6R specific ELISA 48 hours later. F. Expression of primary $m i R-34 a$ (pri-miR-34a) in the indicated cell lines relative to expression in DLD1 cells. 


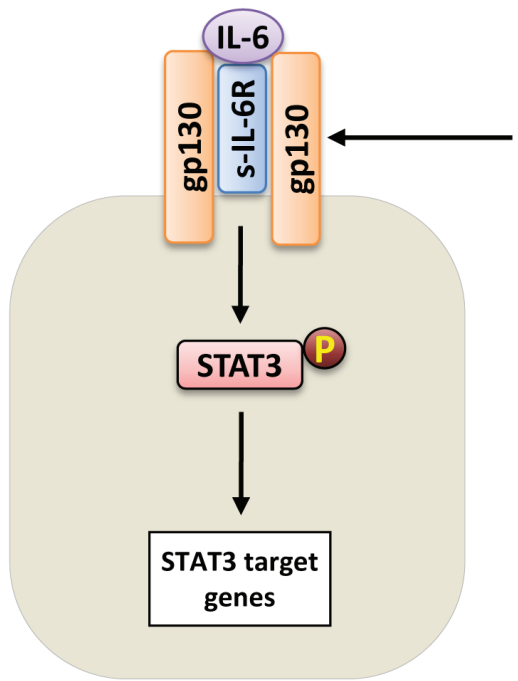

cells not expressing IL-6R

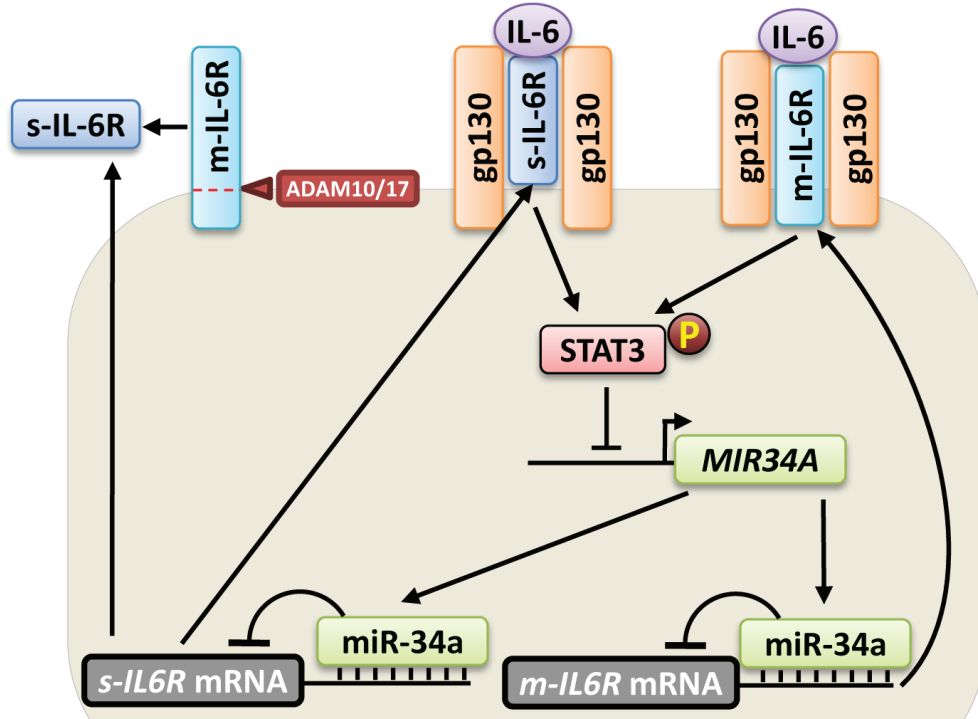

cells expressing $\mathrm{m}-\mathrm{IL}-6 \mathrm{R}$ and $\mathrm{s}-\mathrm{IL}-6 \mathrm{R}$

Figure 4: Effect of miR-34a on m-IL-6R and s-IL-6R mediated signaling. Right side: In cells that express both, m-IL-6R and s-IL-6R, such as macrophages, miR-34a represses the expression of both IL-6R isoforms. Left side: The regulation of s-IL-6R expression by miR-34a does not only influence cells expressing IL-6R, but may also regulate STAT3 signaling in cells that do not express IL-6R and are responsive only to IL-6 trans-signaling, such as smooth muscle cells, endothelial cells, and keratinocytes.

the $s$-IL6R mRNA isoform are directly targeted and repressed by miR-34a. Taken together, our results show that miR-34a represses the expression of $s-I L 6 R$ mRNA and protein. Therefore, our previous findings about the IL6R/STAT3/miR-34a loop [1] may be extrapolated to the s-IL-6R isoform and may have implications for IL-6 transsignaling (Figure 4). MiR-34a mediated repression of s-IL$6 \mathrm{R}$ in cells that produce s-IL-6R, such as macrophages may thus regulate IL-6 JAK/STAT signaling also in cells that do not produce m-IL-6R, such as smooth muscle cells, endothelial cells, and keratinocytes [7]. Accordingly, miR-34a suppression, which is common in cancer cells, and consequent enhanced production of s-IL-6R could activate JAK/STAT signaling in stromal cells of the tumor microenvironment. Finally, miR-34a mimetics may be used to repress s-IL-6R expression and consequently IL-6 trans-signaling, to treat inflammatory diseases and cancer.

\section{MATERIALS AND METHODS}

\section{Cell culture and reagents}

DLD-1, HCT-15, HT29, Caco-2, LS174T, SW480, and SW620 CRC cell lines were maintained in McCoy's 5A Medium (Invitrogen) containing 10\% FBS. Precursor microRNA oligonucleotides (Ambion pre-miR-34a [PM11030]) were transfected at a final concentration of
$25 \mathrm{nM}$ using Lipofectamine 2000 transfection reagent (Invitrogen) according to manufacturer's instructions.

\section{s-IL-6R-specific ELISA}

At indicated time points, conditioned media was collected and subjected to s-IL-6R specific ELISA (R\&D, DR600). The analyses were performed according to manufacturer's instructions. In all experiments equal number of cells was seeded.

\section{RNA isolation and $\mathrm{qPCR}$}

Total RNA was isolated using the Total RNA Isolation Kit (Roche) according to the manufacturer's instructions. cDNA was generated from $1 \mu \mathrm{g}$ total RNA per sample using the Verso cDNA synthesis kit (Thermo Scientific). qPCR was performed by using the LightCycler 480 (Roche) and the Fast SYBR Green Master Mix (Applied Biosystems). mRNA expression was normalized using detection of GAPDH. Results are represented as fold induction using the $\Delta \Delta \mathrm{Ct}$ method [24] with the control set to 1. Oligonucleotides: GAPDH (fwd: 5'-GCTCTCTGCTCCTCCTGTTC-3', rev: 5'-ACGACCAAATCCGTTGACTC-3'), pri-miR$34 a$ (fwd: 5'-CGTCACCTCTTAGGCTTGGA-3', rev: 5'-CATTGGTGTCGTTGTGCTCT-3'), m-IL6R 
(fwd: 5'-CTCCTCTGCATTGCCATTGT-3', rev: 5'- TGTGGCTCGAGGTATTGTCA-3'), s-IL6R (fwd: 5'-CGACAAGCCTCCCAGGTTCA-3', rev: 5'-CGGTTGTGGCTCGAGGTATT-3').

\section{Dual reporter assays}

The 3'-UTR of human $s$-IL6R was PCR amplified from oligo-dT-primed cDNA of human diploid fibroblasts with the Verso cDNA kit (Thermo Scientific), inserted into pGL3-control-MCS, and verified by sequencing. Mutations in the miR-34a seed-matching sequence were generated with the QuikChange Mutagenesis Kit according to the manufacturer's instructions (Stratagene). For luciferase assays, H1299 cells were seeded in 12-well format dishes with $3 \times 10^{4}$ cells/well and transfected after 24 hours with $100 \mathrm{ng}$ of the indicated firefly luciferase reporter plasmid, $20 \mathrm{ng}$ of Renilla reporter plasmid as a normalization control, and $25 \mathrm{nM}$ of miR-34a or a negative control oligonucleotide. After 48 hours, a Dual Luciferase Reporter assay (Promega) was performed according to the manufacturer's instructions. Fluorescence intensities were measured with an Orion II luminometer (Berthold) in 96well format and analyzed with the SIMPLICITY software package (DLR).

\section{ACKNOWLEDGMENTS}

We are grateful to Prof. Stefan Rose-John (ChristianAlbrechts-Universität, Kiel, Germany) for comments on the manuscript. HL is a fellow of the China Scholarship Council. Work in HH's lab is funded by the DFG, Deutsche Krebshilfe, Else-Kröner-Fresenius-Stiftung, Friedrich-Baur-Stfitung and the Rudolf-Barlting-Stiftung.

\section{CONFLICTS OF INTEREST}

There is no conflict of interest.

\section{REFERENCES}

1. Rokavec M, Oner MG, Li H, Jackstadt R, Jiang L, Lodygin D, Kaller M, Horst D, Ziegler PK, Schwitalla S, SlottaHuspenina J, Bader FG, Greten FR, and Hermeking H. IL-6R/STAT3/miR-34a feedback loop promotes EMTmediated colorectal cancer invasion and metastasis. J Clin Invest. 2014; 124: 1853-1867.

2. Scheller J, and Rose-John S. Interleukin-6 and its receptor: from bench to bedside. Med Microbiol Immunol. 2006; 195: 173-183.

3. Rossi JF, Lu ZY, Jourdan M, and Klein B. Interleukin-6 as a Therapeutic Target. Clin Cancer Res. 2015; 21: 1248-1257.

4. Nishimoto N, and Kishimoto T. Interleukin 6: from bench to bedside. Nat Clin Pract Rheumatol. 2006; 2: 619-626.
5. Rose-John S, Waetzig GH, Scheller J, Grotzinger J, and Seegert D. The IL-6/sIL-6R complex as a novel target for therapeutic approaches. Expert Opin Ther Targets. 2007; 11: 613-624.

6. Scheller J, Ohnesorge N, and Rose-John S. Interleukin-6 trans-signalling in chronic inflammation and cancer. Scand J Immunol. 2006; 63: 321-329.

7. Chalaris A, Garbers C, Rabe B, Rose-John S, and Scheller J. The soluble Interleukin 6 receptor: generation and role in inflammation and cancer. Eur J Cell Biol. 2011; 90: 484494.

8. Rose-John S, Scheller J, Elson G, and Jones SA. Interleukin-6 biology is coordinated by membrane-bound and soluble receptors: role in inflammation and cancer. $\mathrm{J}$ Leukoc Biol. 2006; 80: 227-236.

9. Hermeking H. MicroRNAs in the p53 network: micromanagement of tumour suppression. Nat Rev Cancer. 2012; 12: 613-626.

10. Rokavec M, Li H, Jiang L, and Hermeking H. The p53/ miR-34 axis in development and disease. J Mol Cell Biol. 2014; 6: 214-230.

11. Siemens H, Jackstadt R, Hunten S, Kaller M, Menssen A, Gotz U, and Hermeking H. miR-34 and SNAIL form a double-negative feedback loop to regulate epithelialmesenchymal transitions. Cell Cycle. 2011; 10: 4256-4271.

12. Siemens H, Jackstadt R, Kaller M, and Hermeking H. Repression of c-Kit by p53 is mediated by miR-34 and is associated with reduced chemoresistance, migration and stemness. Oncotarget. 2013; 9: 1399-1415.

13. Han Z, Zhang Y, Yang Q, Liu B, Wu J, Yang C, and Jiang Y. miR-497 and miR-34a retard lung cancer growth by coinhibiting cyclin E1 (CCNE1). Oncotarget. 2015;6: 1314963.

14. Ma W, Xiao GG, Mao J, Lu Y, Song B, Wang L, Fan S, Fan P, Hou Z, Li J, Yu X, Wang B, Wang H, et al. Dysregulation of the miR-34a-SIRT1 axis inhibits breast cancer stemness. Oncotarget. 2015; 12: 10432-10444.

15. Xu X, Chen W, Miao R, Zhou Y, Wang Z, Zhang L, Wan Y, Dong Y, Qu K, and Liu C. miR-34a induces cellular senescence via modulation of telomerase activity in human hepatocellular carcinoma by targeting FoxM1/c-Myc pathway. Oncotarget. 2015; 6: 3988-4004.

16. Chen WY, Liu SY, Chang YS, Yin JJ, Yeh HL, Mouhieddine TH, Hadadeh O, Abou-Kheir W, and Liu YN. MicroRNA-34a regulates WNT/TCF7 signaling and inhibits bone metastasis in Ras-activated prostate cancer. Oncotarget. 2015; 6: 441-457.

17. Wang AM, Huang TT, Hsu KW, Huang KH, Fang WL, Yang MH, Lo SS, Chi CW, Lin JJ, and Yeh TS. Yin Yang 1 is a target of microRNA-34 family and contributes to gastric carcinogenesis. Oncotarget. 2014; 5: 5002-5016.

18. Schirmer U, Doberstein K, Rupp AK, Bretz NP, Wuttig D, Kiefel H, Breunig C, Fiegl H, Muller-Holzner E, Zeillinger R, Schuster E, Zeimet AG, Sultmann H, et al. Role of miR- 
$34 \mathrm{a}$ as a suppressor of L1CAM in endometrial carcinoma. Oncotarget. 2014; 5: 462-472.

19. Agostini M, and Knight RA. miR-34: from bench to bedside. Oncotarget. 2014; 5: 872-881.

20. Siemens H, Neumann J, Jackstadt R, Mansmann U, Horst D, Kirchner T, and Hermeking H. Detection of miR34a promoter methylation in combination with elevated expression of c-Met and beta-catenin predicts distant metastasis of colon cancer. Clin Cancer Res. 2013; 19: 710720.

21. Carstens JL, Lovisa S, and Kalluri R. Microenvironmentdependent cues trigger miRNA-regulated feedback loop to facilitate the EMT/MET switch. J Clin Invest. 2014; 124: 1458-1460.

22. Matsuo K, Oka M, Murase $\mathrm{K}$, Soda $\mathrm{H}$, Isomoto $\mathrm{H}$, Takeshima F, Mizuta Y, Murata I, and Kohno S. Expression of interleukin 6 and its receptor in human gastric and colorectal cancers. J Int Med Res. 2003; 31: 69-75.

23. Rose-John S. IL-6 trans-signaling via the soluble IL-6 receptor: importance for the pro-inflammatory activities of IL-6. Int J Biol Sci. 2012; 8: 1237-1247.

24. Pfaffl MW. A new mathematical model for relative quantification in real-time RT-PCR. Nucleic Acids Res. 2001; 29: 2002-2007. 